\title{
Charmed spectroscopy from a nonperturbatively determined relativistic heavy quark action in full QCD
}

\section{Huey-Wen Lin* for the RBC Collaboration}

Department of Physics, Columbia University, New York, NY 10027

Thomas Jefferson National Accelerator Facility, Newport News, VA 23606

E-mail: hwlinejlab.org

\begin{abstract}
We present a preliminary calculation of the charmed meson spectrum using the $2+1$ flavor domain wall fermion lattice configurations currently being generated by the RBC and UKQCD collaborations. The calculation is performed using the 3-parameter, relativistic heavy quark action with nonperturbatively determined coefficients. We will also demonstrate a step-scaling procedure for determining these coefficients nonperturbatively using a series of quenched, gauge field ensembles generated for three different lattice spacings.
\end{abstract}

XXIV International Symposium on Lattice Field Theory

July 23-28 2006

Tucson Arizona, US

${ }^{*}$ Speaker. 


\section{Introduction}

Flavor physics and CP violation play an important role in particle physics. Lattice QCD provides a first-principles approach for probing these interesting physics topics starting from the Standard Model. However, in the application to heavy quark physics, $(m a) \ll 1$ is no longer true and $(m a)^{n}$ terms become significant. Because $a$ must be made very small, direct simulation of heavy quarks by brute force becomes too expensive. Thus, an effective theory is needed to carry out the calculation. There are multiple fermion actions being used in lattice calculations; see reviews in Refs. [1, 2, 3].

In this work, we will concentrate on the effective theory called relativistic heavy quark (RHQ) action [4, 5, 6, 7], as

$$
S=\sum_{n} \bar{\psi}_{n}\left\{m_{0}+\gamma_{0} D_{0}-\frac{1}{2} a D_{0}^{2}+\zeta\left[\vec{\gamma} \cdot \vec{D}-\frac{1}{2} a(\vec{D})^{2}\right]-\sum_{i} \frac{i}{4} c_{\mathrm{P}} a \sigma_{\mu v} F_{\mu v}\right\} \psi_{n} .
$$

We specifically use the formulation proposed in Refs. [6, 7]. The main idea is that in the heavy quark case, the temporal covariant derivative $D_{0}$ is around the order of $m a$ and should not be treated the same way as the spatial derivatives $D_{i}$; we refer to this method as "RHQ" power counting. Following the Symanzik improvement procedure, we found that there are only three necessary parameters in the action: $m_{0}, \zeta, c_{\mathrm{P}}$. The advantages of using this action are that it

- Systematically absorbs mass factors into the coefficients

- Has small cutoff effects: $\left(\Lambda_{\mathrm{QCD}} a\right)^{2}$ for heavy-light systems and $\left(\alpha_{s} m a\right)^{2}$ for onium systems

- Goes to Sheikoleslami-Wohelert (SW) action when $m a \ll 1$ and Non-Relativistic QCD when $m a \gg 1$

In high-precision calculations, we must first determine the correct action parameters before using the action to calculate observables. Since perturbation theory introduces error into our parameter calculation, a nonpertubatively determined action is important.

\section{Nonpertubatively determined RHQ action}

There are various ways of determining the action coefficients. One approach that has been widely used is lattice perturbation theory. However, unlike continuum perturbation theory, LPT does not converge quickly, and it has errors that are hard to control. Another approach is to tune action parameters by matching physical observables sensitive to particular parameters to their known experimental values. However, we would lose some of the predictive power of our theory. Last but not least, we may use a step-scaling technique[8]; instead of using brute force to directly simulate the heavy quark on a fine lattice (where $m a \ll 1$ ) in a large box, we connect fine lattices with small physical volumes to coarser lattices with larger physical volumes, as shown in the Figure 1. This is computationally much cheaper than the brute-force calculation.

We can apply the step-scaling technique to determine the coefficients in the action by using

1. off-shell quantities: The quark-gluon vertex is a candidate for determining the coefficients coupling to the Sheikholeslami and Wohlert term. We will be able to use the off-shell improvement to determine not only the coefficients in the action, but also the $O(a)$ off-shell improvement coefficients in the quark field, which allows us to apply nonperturbative renormalization (NPR) directly[9, 10]. However, this requires many more parameters to be determined numerically and involves subtle, gauge-non-invariant terms to be added to the action and operators. 


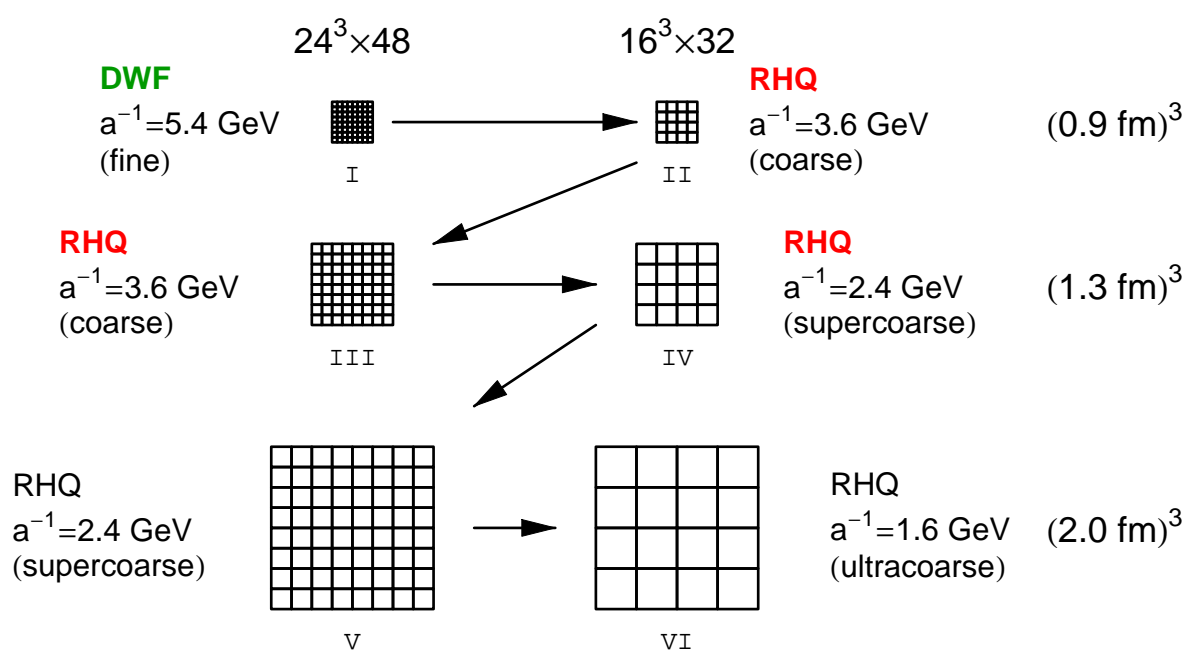

Figure 1: The sequence of lattice sizes and lattice spacings used to determine the coarse-lattice heavy quark parameters through a step-scaling technique beginning with a comparison with an $O(a)$-improved light quark calculation: The matchings between the top four lattice spacing-volume combinations are described in this work.

2. on-shell quantities: We use mass combinations of pseudoscalar (PS), vector (V), scalar (S) and axial-vector (AV) mesons in heavy-heavy $(h h)$ and heavy-light $(h l)$ systems computed in fixed, finite volume[11].

(a) spin-averaged: $m_{\mathrm{sa}}^{h h}=\frac{1}{4}\left(m_{\mathrm{PS}}^{h h}+3 m_{\mathrm{V}}^{h h}\right), m_{\mathrm{sa}}^{h l}=\frac{1}{4}\left(m_{\mathrm{PS}}^{h l}+3 m_{\mathrm{V}}^{h l}\right)$

(b) hyperfine splitting: $m_{\mathrm{hs}}^{h h}=m_{\mathrm{V}}^{h h}-m_{\mathrm{PS}}^{h h}, m_{\mathrm{hs}}^{h l}=m_{\mathrm{V}}^{h l}-m_{\mathrm{PS}}^{h l}$

(c) spin-orbit averaged and splitting: $m_{\mathrm{soa}}^{h h}=\frac{1}{4}\left(m_{\mathrm{S}}^{h h}+3 m_{\mathrm{AV}}^{h h}\right), m_{\mathrm{sos}}^{h h}=m_{\mathrm{AV}}^{h h}-m_{\mathrm{S}}^{h h}$

(d) dispersion relation: $E^{2}=m^{2}+c^{2} p^{2}$.

The simulation was carried out on a QCDOC 512-node machine at $420 \mathrm{MHz}$ clock frequency. We used quenched Wilson gauge action with the heatbath algorithm, taking 20,000 sweeps for thermalization and measuring 100 configurations at 10,000 sweeps separation. Coulomb gaugefixed hydrogenic source smearing is used to improve overlap with ground states. We use a linear ansatz relating the action coefficients $\left(X_{\mathrm{RHQ}}\right)$ and the corresponding measurements $\left(Y_{\text {coarse }}^{i, d}\right)$ :

$$
Y_{\text {coarse }}^{i, d}=A^{d}+J^{d} \cdot X_{\mathrm{RHQ}}^{i},
$$

where $J$ and $A$ can be obtained from either fitting parameters or using finite differences directly from a Cartesian set. These two approaches agree within errors. The detailed choices of parameters, lattice spacing determinations and analysis can be found in Ref. [11, 12]. The RHQ coefficients for the $a^{-1}=2.4 \mathrm{GeV}$ lattice, after two stages of matchings starting from the $a^{-1}=5.4 \mathrm{GeV}$ lattice, are

$$
\begin{aligned}
c_{\mathrm{P}}\left(m_{0}\right) & =1.65(3)+0.12(6) m_{0}+1.06(4) m_{0}^{2} \\
\zeta\left(m_{0}\right) & =1.090(10)+0.318(16) m_{0}-0.092(10) m_{0}^{2} .
\end{aligned}
$$

We simulate at three charm quark points from the RHQ coefficients, $\left(X_{\mathscr{C}}^{(3)}\right)^{T}=\left\{m_{0}, c_{\mathrm{P}}, \zeta\right\}=$ $\{-0.06106,1.651,1.070\},\{0.02173,1.653,1.097\},\{0.1086,1.686,1.122\}$ respectively. The spin-averaged mass $\left(m_{\overline{1 S}}=\left[m_{\eta_{c}}+3 m_{J / \Psi}\right] / 4\right)$ is used to determine the bare charm quark mass. Figure 2 shows a summary of our results along with the experimental values, with statistical errors 


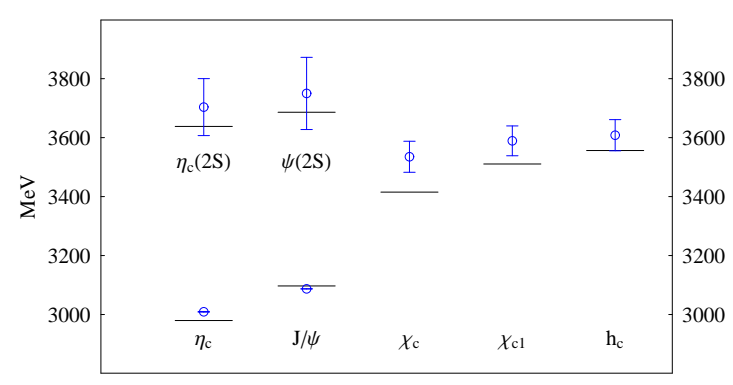

Figure 2: The spectrum of the charmonium system: The circles are our results with statistical errorbars and the horizontal lines correspond to experimental values.

only. All $P$-wave masses are within one standard deviation higher than the experimental one. The spin-orbit splitting is $54(33) \mathrm{MeV}$, similar to what we observed in the past; it is a difficult quantity to determine precisely. The hyperfine splitting is $77.8(15) \mathrm{MeV}$, about $40 \%$ smaller than the experimental splitting $(116 \mathrm{MeV})$. The hyperfine splitting is typically not given correctly in lattice calculations. A list of quenched hyperfine splittings calculated from RHQ lattice QCD is given in Figure 3. The hyperfine splitting from the one-loop Tsukuba approach[13] ranges from 75.7 to $64.6 \mathrm{MeV}$ using a fixed volume of $(1.8 \mathrm{fm})^{3}$ with lattice spacing varying from 0.0562 to 0.112. Our hyperfine splitting at the same lattice spacing is about $10 \%$ higher than the one-loop quenched result[13], which is encouraging. We might be able to resolve the hyperfine splitting problem when we apply our nonperturbative approach in a full-QCD calculation.

For the excited states, we use an additional smearing function on the heavy quark field,

$$
\Psi_{\mathrm{exc}}(r)=\left(1-r / 2 r_{0}\right) e^{-r / 2 r_{0}},
$$

to improve the overlap with excited states. Then, we perform a four-parameter double-cosh fit to extract the ground and excited states. The radial excited states from our low-statistics data appear consistent with experiment, as does the $\overline{2 S}-\overline{1 S}$ splitting. This measurement can be easily improved with more configurations and a constrained fit.

So far in this discussion, we have been taking the lattice spacing from the static quark potential with scale determined by Sommer scale $r_{0}=0.5 \mathrm{fm}$ from phenomenological models. We could also determine the lattice spacing using the $\overline{1 P}-\overline{1 S}$ splitting in the charmonium system. Here we adopt the singlet $1 P$ state, $m_{h_{c}}$, for the $\overline{1 P}$ mass. Recalculating the bare charm quark mass and the lattice spacing, we get $a m_{c}=0.15599(14)$ and $a^{-1}=2.24(14) \mathrm{GeV}$. This suggests $r_{0}=0.534(33) \mathrm{fm}$. This is close to what we assumed in the previous scale determination. From the statistics we have, we do not observe problems due to determining the lattice spacing in this calculation. Thus, we will continue use $a^{-1}=2.4 \mathrm{GeV}$ for the rest of this work.

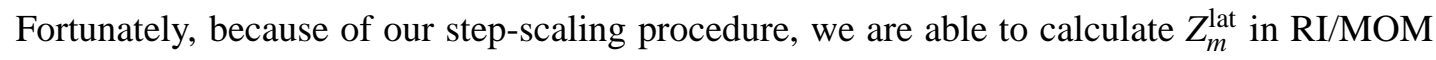
scheme[9] using DWF on the fine lattice and to include an estimate of finite-mass effects $\left(\sim\left(\operatorname{am}_{h}\right)^{2}\right)$ in the systematic error for the final calculation. We calculate the quark propagator and we expect that in the large-momentum region the scalar part of the quark propagator behaves like[14]

$$
\frac{1}{12} \operatorname{Tr}\left(S_{L}^{-1}(p)\right)=C \times p^{2}+Z_{m} Z_{q}\left(m+m_{\mathrm{res}}\right)+\frac{B}{p^{2}}+O\left(p^{-4}\right) .
$$

When this equation is evaluated, we find the renormalized mass $m_{c}^{\mathrm{RI}}=1.124(9) \mathrm{GeV}$ in RI/MOM 


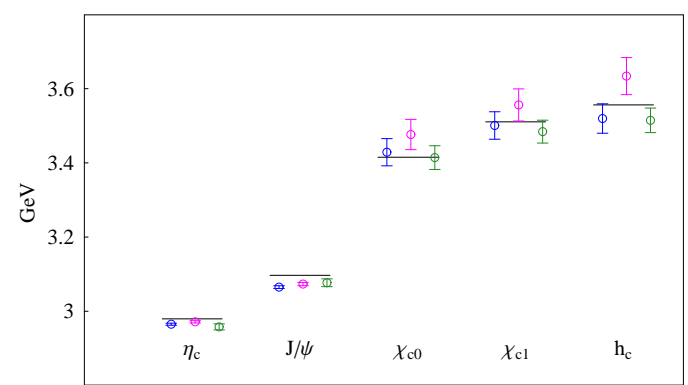

Figure 4: The spectrum of the charmonium system from NP RHQ in full QCD: The blue, purple and green correspond to lattices with $a m_{\text {sea }}=$ $0.01,0.02,0.03$ respectively, and the horizontal lines correspond to experimental values.

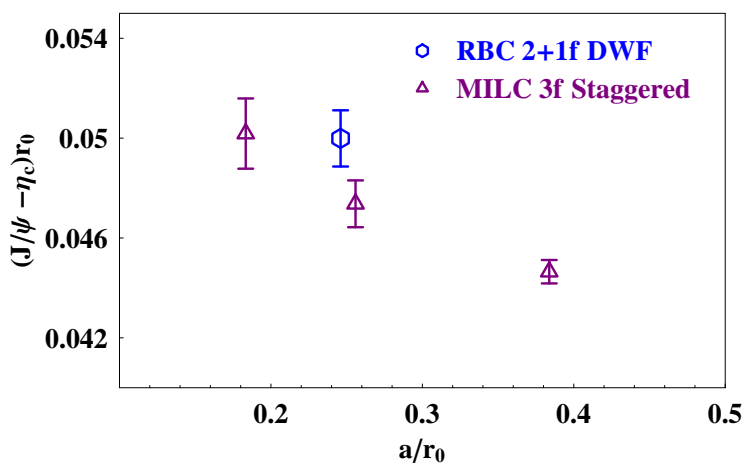

Figure 5: A comparison of hyperfine-splitting from our $a m_{\text {sea }}=0.01$ and from MILC 2+1 dynamical calculations.

scheme. We matched RI/MOM scheme to $\overline{\mathrm{MS}}$ scheme and obtained the renormalization-groupinvariant mass[15], $m^{\mathrm{RGI}}=2.401(21) \mathrm{GeV}$. We find at the scale of averaged $m_{c}$, our renormalized charm quark mass is $1.314(11) \mathrm{GeV}$. This statistical error is determined by applying the jackknife method to the supercoarse statistical ensemble and then inflating the result by a factor of $\sqrt{3}$ to include the statistical errors introduced at the other two matching stages; thus, $m_{c}^{\overline{\mathrm{MS}}}\left(\bar{m}_{c}\right)=$ $1.314(18) \mathrm{GeV}$.

\section{Full QCD}

The RBC and UKQCD collaborations have generated $2+1$ flavor dynamical DWF ensembles, at fixed lattice spacing $a^{-1} \approx 1.6 \mathrm{GeV}$ (set by Sommer scale $0.5 \mathrm{fm}$ ), with two volumes, $\approx 2^{3}$ and $3^{3} \mathrm{fm}^{3}$, using Iwasaki gauge action $(\beta=2.13)$. The up and down sea quark mass in terms of the inverse lattice spacing are $0.01,0.02$ and 0.03 with the strange quark mass set to 0.04 . We first apply the NP RHQ coefficients directly on 2+1-flavor dynamical configurations to check out the heavy-heavy sector of charm physics, where the small volume lattice is sufficient. The results are shown in Figure 4 for three different sea quark masses, labeled by blue, purple and green as the mass increases, with statistics of 75 configurations. First, we can see that there is an instant boost in the value of the hyperfine splitting between quenched lattices and dynamical ones, and the $P$-wave states all agree with the experiment values within statistics error bars. In Figure 5, we compare our hyperfine splitting with the calculation obtained from MILC[16]. Since MILC and RBC/UKQCD use different reference scales, Sommer scale $r_{0}$, to set the lattice spacing and the hyperfine splitting is sensitive to this reference scale, we plot the dimensionless quantity (the product of hyperfine splitting and $r_{0}$ ) as a function of $a / r_{0}$. We noted that even though our lattice is coarser than the MILC ones, our hyperfine value is consistent with their finest point. This is a very encouraging result to test on our NP coefficients. However, since the gauge actions used in the quenched and dynamical simulations are not matched, there might be $O\left(a^{2}\right)$ effects in the gauge action. Thus, our hyperfine splitting can be further improved if we carry out the NP RHQ project dynamically.

Carrying out a step-scaling calculation in full QCD is very expensive and time-consuming; in the meantime, we can match the RHQ coefficients on the RBC/UKQCD full QCD lattices to the experimental charmonium spin-averaged mass and spin-splitting mass and dispersion relation. This is accurate through $|\vec{p} a|$ and to all order of $(m a)^{n}$. Therefore, we can immediately start studying 


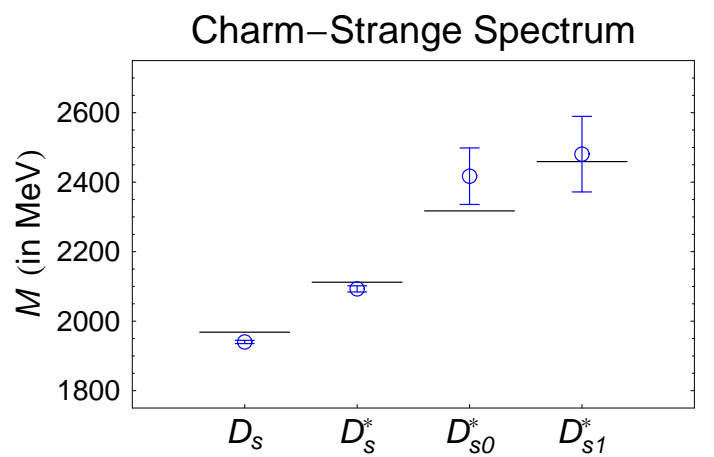

Figure 6: (Left) The spectrum of $D_{s}$ on the $m_{\text {sea }}=$ 0.01 ensemble with $m_{\text {strange }}=0.04$.

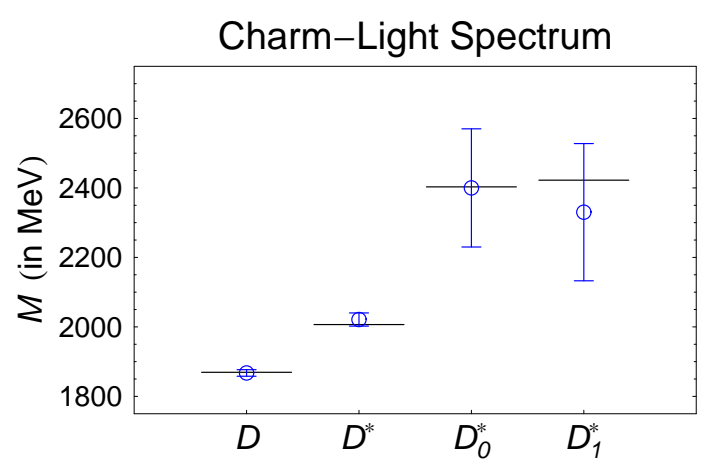

Figure 7: (Right) The spectrum of $D$ on the $m_{\text {sea }}=$ 0.01 ensemble with $m_{\text {up }, \text { down }}=0.01$.

other charmed states, such as charmed baryons, charm-light systems, charmonium excited states (including exotic ones). We first demonstrate on our smallest sea quark mass configurations; we obtained coefficients in the action

$$
X_{\mathrm{RHQ}}=\left\{m_{0}, c_{\mathrm{P}}, \zeta\right\}=\{0.46(4), 2.50(9), 1.285(19)\} .
$$

Again, the bare charm mass is set by the experimental charmonium spin-averaged mass. We use 126 configurations. Due to time constraints, here we only obtain results for the charm-light system, as shown in Figure 6 and 7 . Both spectra are consistent with experimental values. The $S$-wave splittings are:

$$
\begin{aligned}
& m_{D^{*}}-m_{D}=154(16) \mathrm{MeV} \\
& m_{D_{s}^{*}}-m_{D_{s}}=153(7) \mathrm{MeV},
\end{aligned}
$$

which are consistent with the experimental values of $142 \mathrm{MeV}$ and $144 \mathrm{MeV}$ respectively.

\section{Conclusion}

In this work, we have shown that the three coefficients $\left(m_{0}, c_{\mathrm{P}}\right.$ and $\left.\zeta\right)$ for the RHQ action can be determined nonperturbatively with step scaling. We demonstrate some interesting quenched physics with improvement of the hyperfine splitting in charmonium. It also allows us to determine the charm quark mass: $m_{c}^{\overline{\mathrm{MS}}}\left(\bar{m}_{c}\right)=1.314(18) \mathrm{GeV}$, without complicated off-shell improvement on the RHQ action. This makes future application to full QCD feasible. The direct application of NP quenched coefficients to dynamical configurations gives promising charmonium features such as better hyperfine splitting than existing dynamical results. We further tune the RHQ action with experimental numbers and produce promising results for the charmonium and charm-light systems. In the near future, we would also like to calculate more meson states involving derivative operators (including exotic ones) and also to study charmed baryons masses. It should also be easy to apply this method to the $B$ system. In the long term, we would like to carry on the step-scaling technique first to the NP determined RHQ action in full QCD and then to decay constants and form factors involving one or two heavy quarks with RI/MOM NPR.

\section{Acknowledgements}

We acknowledge helpful discussions with Tanmoy Bhattacharya, Peter Boyle, Paul Mackenzie, Andreas Kronfeld, Sinya Aoki, Yoshinobu Kuramashi and members of the RBC collaboration. In addition, we thank Peter Boyle, Dong Chen, Mike Clark, Norman Christ, Calin Cristian, Zhihua Dong, Alan Gara, Andrew Jackson, Balint Joo, Chulwoo Jung, Richard Kenway, Changhoan Kim, 
Ludmila Levkova, Xiaodong Liao, Guofeng Liu, Robert Mawhinney, Shigemi Ohta, Konstantin Petrov, Tilo Wettig and Azusa Yamaguchi for developing the QCDOC machine and its software. This development and the resulting computer equipment used in this calculation were funded by the U.S. DOE grant DE-FG02-92ER40699, PPARC JIF grant PPA/J/S/1998/00756 and by RIKEN.

This work was supported by DOE grant DE-FG02-92ER40699, PPARC grant PP/C504386/1 and PPARC grant PP/D000238/1. We thank RIKEN, BNL and the U.S. DOE for providing the facilities essential for the completion of this work.

\section{References}

[1] A. S. Kronfeld, Heavy quarks and lattice QCD, Nucl. Phys. Proc. Suppl. 129 (2004) 46-59, hep-lat/0310063.

[2] M. Wingate, Status of lattice flavor physics, Nucl. Phys. Proc. Suppl. 140 (2005) 68-77, hep-lat/0410008.

[3] M. Okamoto, Full determination of the ckm matrix using recent results from lattice QCD, PoS LAT2005 (2006) 013, hep-lat/0510113.

[4] A. X. El-Khadra, A. S. Kronfeld, and P. B. Mackenzie, Massive fermions in lattice gauge theory, Phys. Rev. D55 (1997) 3933-3957, hep-lat/9604004.

[5] S. Aoki, Y. Kuramashi, and S.-i. Tominaga, Relativistic heavy quarks on the lattice, Prog. Theor. Phys. 109 (2003) 383-413, hep-lat/0107009.

[6] N. H. Christ, M. Li, and H.-W. Lin, Relativistic heavy quark effective action, hep-lat/0608006.

[7] N. H. Christ, M. Li, and H.-W. Lin, Improved heavy quark action, PoS LAT2006 (2006) 171.

[8] M. Luscher, S. Sint, R. Sommer, and H. Wittig, Non-perturbative determination of the axial current normalization constant in $O($ a) improved lattice QCD, Nucl. Phys. B491 (1997) 344-364, hep-lat/9611015 .

[9] G. Martinelli, C. Pittori, C. T. Sachrajda, M. Testa, and A. Vladikas, A general method for nonperturbative renormalization of lattice operators, Nucl. Phys. B445 (1995) 81-108, hep-lat/9411010 .

[10] H.-W. Lin, Quark-gluon vertex with an off-shell $O($ a $)$-improved chiral fermion action, Phys. Rev. D73 (2006) 094511, hep-lat/0510110 .

[11] H.-W. Lin and N. Christ, Non-perturbatively determined relativistic heavy quark action, hep-lat/0608005.

[12] H.-W. Lin, Charm physics with a nonperturbatively determined relativistic heavy quark action, Ph.D. thesis (2006).

[13] CP-PACS Collaboration, Y. Kuramashi et al., Quenched scaling study of charm and bottom systems with a relativistic heavy quark action, PoS LAT2005 (2006) 226.

[14] T. Blum et al., Non-perturbative renormalisation of domain wall fermions: Quark bilinears, Phys. Rev. D66 (2002) 014504, hep-lat/0102005 .

[15] K. G. Chetyrkin and A. Retey, Renormalization and running of quark mass and field in the regularization invariant and ms-bar schemes at three and four loops, Nucl. Phys. B583 (2000) 3-34, hep-ph/9910332 .

[16] S. Gottlieb et al., Onium masses with three flavors of dynamical quarks, PoS LAT2005 (2006) 203, hep-lat/0510072 . 